\title{
Efficient algorithm for boson sampling with partially distinguishable photons
}

\author{
J.J. Renema, A. Menssen, W.R. Clements, G. Triginer, W.S. Kolthammer and I.A. Walmsley \\ Clarendon Labs, Department of Physics, Oxford University, Parks Road OX1 3PU Oxford
}

\begin{abstract}
We demonstrate how boson sampling with photons of partial distinguishability can be expressed in terms of interference of fewer photons. We use this observation to propose a classical algorithm to simulate the output of a boson sampler fed with photons of partial distinguishability. We find conditions for which this algorithm is efficient, which gives a lower limit on the required indistinguishability to demonstrate a quantum advantage. Under these conditions, adding more photons only polynomially increases the computational cost to simulate a boson sampling experiment.
\end{abstract}

Boson sampling [1] provides a promising route towards demonstrating a quantum advantage, i.e. a computation by a quantum system that exceeds what is possible with a classical one. In boson sampling, the task is to provide a sample from the output of a linear transformation of optical modes, some of which are fed with single photons. For a sufficient number of photons and modes, a suitable quantum machine directly implementing this problem will outperform a realistic classical computer simulating the experiment. This result has spurred a range of experimental efforts 2 [8].

A crucial challenge for computational problems based on boson sampling is to accommodate imperfections that arise in real-world devices. An essential aspect of the original proposal [1] was to show that for small deviations from the ideal machine, the achieved sampling problem retains computational hardness. However, descriptions of experiments that incorporate realistic models of photon distinguishability [9] or beam-splitter deviations [10] are unable to meet this error requirement. There is therefore a need to devise photon sampling problems with improved error tolerance.

There exist two approaches to demarcating the line between viable and non-viable extensions of boson sampling. In the top-down approach, the original complexity proof is extended 'downwards', showing that the resulting output distribution for systems of increasing imperfection is still hard to sample from. This approach has had some success: for example, it has been shown that the issue of unreliable single photon generation can be overcome, and that the hardness of the resulting 'scattershot' boson sampling problem is equivalent to that of the original boson sampling problem [11, 12].

The alternate bottom-up approach is to construct new efficient classical algorithms for boson samplers with particular imperfections. This approach leads to constructive proofs that rule out a computational advantage, thereby showing performance limits that must be exceeded by quantum machines. For example, RahimiKeshari et al [13] used generalizations of the Wigner function as a way to construct a classical algorithm which can efficiently simulate certain lossy boson samplers.

In this work, we consider which-way information of the interfering photons as an imperfection that compromises

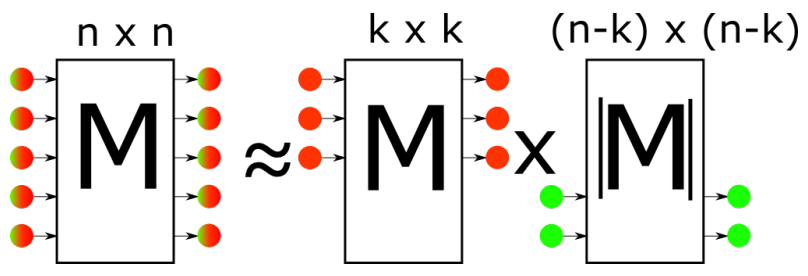

Figure 1. A pictorial representation of our result. We show that boson sampling with $n$ photons of partial distinguishability (represented by the mixed red-green balls) can be approximated as computing the outcome of a series of smaller permanents of size $k$, combined with probabilistic transmission of the remaining $n-k$ photons. The value of $k$ at which this approximation works is set by the value of the distinguishability.

the hardness of a boson sampler. We show a classical algorithm that efficiently approximates detection probabilities in the limit of many photons, given the photons have some partial distinguishability. We then use this algorithm to consider problems of finite size and estimate a level of indistinguishability that must be surpassed to demonstrate of quantum advantage. The basis of our algorithm is, schematically depicted in Figure 1, that for partially distinguishable photons, the probability of a given outcome can be approximated by terms that involve fewer interfering photons, where the remaining ones do not interfere at all. For a given error tolerance and indistinguishability, we determine a number of photons above which this approach succeeds while requiring only a polynomial increase in the computational steps as the number of photons increases further. We use this result to estimate a lower bound on the photon quality required to demonstrate a quantum advantage: for 50 photons and an error threshold of $10 \%$, the degree of indistinguishability of the interfering photons must be higher than $94.7 \%$.

Multiphoton interference at partial distinguishability has been studied extensively [9, 14 21]. For boson sampling with fully indistinguishable photons, the probability of a particular detection outcome is given by $P=$ $|\operatorname{Perm}(M)|^{2}$, where $M$ is a submatrix of the unitary $U$, where the rows and columns of $M$ are chosen to correspond to the input and output modes of interest, respectively. In this work, we will use the formalism of Tichy 
[16], where the probability of a particular detection outcome (i.e. photons emerging at particular outputs) is:

$$
P=\sum_{\sigma \in \Sigma}\left(\prod_{j} S_{\sigma_{j} j}\right) \operatorname{Perm}\left(M * M_{1, \sigma}^{*}\right),
$$

where $M$ a submatrix constructed in the same way as for fully indistinguishable boson sampling, $*$ denotes the elementwise product, and $M^{*}$ is the elementwise complex conjugation. The notation $M_{1, \sigma}$ indicates that the rows of $M$ are unpermuted, and that the columns are permuted according to $\sigma$, and we will use this notation convention throughout. The matrix of mutual distinguishabilities $S$ is given by $S_{i j}=\left\langle\Psi_{i} \mid \Psi_{j}\right\rangle$, where $\Psi_{i}$ is the $i$-th single-photon wavefunction. The set of permutations of size $n$ is denoted $\Sigma$.

There are two extreme cases to note. First, if $S_{i j}=1$, eq. 1 reduces to the standard expression for boson sampling with fully indistinguishable particles. For $S_{i j}=\delta_{i j}$, eq. 1 reduces to $P=\operatorname{Perm}\left(|M|^{2}\right)$, which is the expression for boson sampling with distinguishable photons. In this latter case, multiphoton interference is absent and the total probability is expressed in terms of singlephoton transmission probabilities instead of transmission amplitudes. Since this matrix contains only positive elements, it can be evaluated to within a multiplicative error in polynomial time [22]. In our work, we will interpolate between these cases, and parameterize the mutual distinguishabilities by a single parameter $x$, with $S_{i j}(x)=x+(1-x) \delta_{i j}$. We will argue at the end of our work that our results apply to more general forms of $S$.

The observation that underlies our work is that the degree of quantum interference in each term in eq. 1 is determined by the number of fixed points (invariant elements) in the corresponding permutation. Each fixed point in $\sigma$ causes the corresponding row from $M$ to enter into the permanent as the modulus squared, meaning that in that term, the corresponding photon does not exhibit interference. Therefore, the size of the matrix of complex elements which must be computed to evaluate each term is set by the number of non-invariant elements in the permutation [16, 17]. Furthermore, terms with many fixed points have a larger weight in the sum: for each element in the permutation which is not a fixed point, the product in eq. 1 will pick up a factor $x<1$ from the off-diagonal elements of $S$ [9, 15]. One can therefore construct a series of succesively more accurate approximations by grouping the terms in eq. 1 by number of fixed points, and then truncating the sum at some value, which we designate $k$. The resulting approximation $P_{k}$ is given by:

$$
P_{k}=\sum_{j=0}^{k} \sum_{\sigma^{j}} x^{j} \operatorname{Perm}\left(M * M_{1, \sigma}^{*}\right)
$$

where we have introduced the notation $\sigma^{j}$ to denote those permutations which have $n-j$ elements as fixed points. To simplify eq. 2 , we note that this permanent, $n-j$ columns will be left unpermuted, and will therefore end up as the modulus squared of the elements. Expanding eq. 2 in all the permuted columns and combining terms, we can separate the permanents of permuted and unpermuted rows as:

$$
P_{k}=\sum_{j=0}^{k} \sum_{\sigma^{j}} x^{j} \sum_{\rho} \operatorname{Perm}\left(M_{\rho, 1} * M_{\rho, \sigma_{p}}^{*}\right) \operatorname{Perm}\left(\left|M_{\bar{\rho}, \sigma_{u}}\right|^{2}\right),
$$

where $\rho$ denotes the $\left(\begin{array}{c}n \\ j\end{array}\right)$ possible combinations of $j$ columns from the matrix $M, \bar{\rho}$ denotes the complemenary rows, and $\sigma_{p}$ and $\sigma_{u}$ denote the permuted and unpermuted elements of $\sigma$, respectively.

We note that the lower the value of $j$, the easier the terms are to compute, since the second of these two permanents can be efficiently evaluated, and the first permanent is of size $j$. The term with $j=0$ represents the case where the photons are treated as fully distinguishable particles. The next term $(j=2)$ represents the firstorder correction, where interference between each pair of photons is considered, and similarly for higher values of $j$. The sequence of $P_{k}$ is therefore ordered by computational cost as well as by degree of quantum interference.

The rest of this paper is dedicated to investigating the properties of these succesive approximations. We start by investigating these approximations numerically, which will lead to some conjectures regarding the scaling behaviour of these approximations with $n$ and $k$, which we will then confirm through more rigorous analysis.

In Figure 2, we numerically investigate the quality of this approximation for a 5-photon boson sampling experiment. We simulate 10.000 Haar-random unitary matrixes of size $N=100$, from which we take the first $n$ modes as input and output without loss of generality, and computed $P_{k}$ for values of $k$ from 1 to 4 . Note that the case $k=1$ only encompasses the identity permutation $(j=0)$, which corresponds to sampling with distinguishable photons. We plot the relative error of our approximation, defined as $\Delta P_{k} / P_{0}$, where $\Delta P=\left|P_{k}-P\right|$ and $P_{0}=n ! / N^{n}$ is the characteristic scaling in the number of photons. For photons with indistinguishability $x=0.9$, which corresponds roughly to a recent demonstration of five-photon boson sampling from quantum dots [8], the approximation $k=4$ already gives an approximation with an error of $10 \%$.

The inset shows how the relative error scales with the photon number $n$. We plot $\Delta P_{2}$ as a function of the photon number $n$. We find that the relative error saturates at moderate values of $n$. This numerical result suggests that in the limit of large photon numbers, the accuracy of our approximation does not depend on the number of 


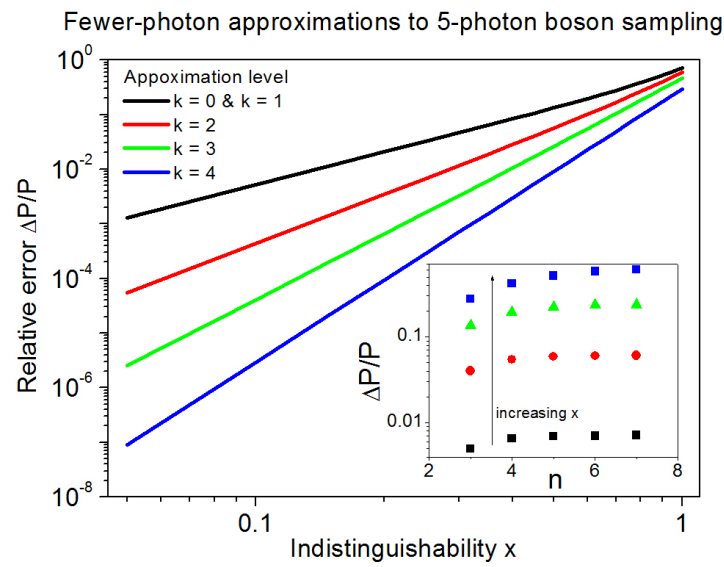

Figure 2. Relative error $\Delta P_{k} / P(x=0)$, where $P$ is the probability of observing some outcome of the boson sampler, as a function of indistinguishability, for different values of $k$. Inset scaling of the relative error with overall photon number for $x=0.25,0.5,0.75$ and 0.95 (from bottom to top).

photons, i.e. $\Delta P_{k} / P_{0}$ is a function of $k$ and $x$, but not of $n$ if $n$ is sufficiently large. This means that for large enough $n$, if our approximation $P_{k}$ satisfies a given error threshold for a particular value of $k$, that approximation will also work for larger photon numbers. Since the size of the complex-valued permanents is given by $k$, this suggests that adding more photons does not significantly add to the cost of simulating the problem.

We now proceed to prove that this is indeed the case. To do so this, we show two things. First, we need to show that our approximation is efficient, in the sense that adding more photons only induces a polynomial increase in the number of computations required. Second, we need to show that the intuition we obtained from the numerics above is correct, and that the relative error on our approximation does not increase when $n$ is increased.

We begin with the first task: counting the number of terms in eq. 3. The number of terms in the middle of the three sums of eq. 3 is given by the the rencontres number $R_{n, n-j}=\left(\begin{array}{c}n \\ j\end{array}\right) !(j)$, where $! j=\lfloor j ! / e\rceil$ is the subfactorial, which counts the number of permutations which leave $n-j$ elements invariant and scales as $n^{j}$. For each term in the middle sum, we have to evaluate $\left(\begin{array}{c}n \\ j\end{array}\right)$ permanents of size $j$, in the inner sum, which is a quantity which also grows polynomially in $n$. The total number of complex-valued permanents which we are required to compute for particular value of $j$ in the outer sum is therefore $\left(\begin{array}{c}n \\ j\end{array}\right)^{2} !(j)$. The problem therefore scales as $n^{2 j}$, and when we truncate the terms at $k$, the number of terms is therefore of the order of $n^{2 k}$. Using Ryser's algorithm [23] to evaluate each complex-valued perma- nent takes $2^{k} k$ steps, and the whole algorithm scales as $n^{2 k} 2^{k} k$, which scales polynomially in $n$ as required, provided the choice of $k$ needed to satisfy some error bound does not depend on $n$.

Therefore, we now consider the accuracy of the scheme. We write $P$ as a polynomial in $x: P(x)=\sum c_{j} x^{j}$, where the coefficients are given by eq. 3 . We can therefore estimate the error $\Delta P_{k} / P_{0}$ by computing the error term $\sum_{j=k+1}^{n} c_{j}$. We find that the increase in the number of terms with $j$ is precisely balanced out by the decrease in the magnitude of each term (see supplemental material), and that these coefficients are given by:

$\left|c_{j}\right| \approx \sqrt{\left(\sum_{k=0}^{n-j}(1 / k !)\right) R_{n, n-j}\left(\begin{array}{c}n \\ j\end{array}\right) j !(n-j) ! / 2 N^{n} \approx n ! / 2 N,}$

where in the final step we have taken the limit of large $n$. It should be noted that this latter expression does not depend on $j$, and differs by a factor $1 / 2$ from the expectation value of fully distinguishable boson sampling. As an illustration, Figure 3 shows estimates of the absolute values of the coefficients $|c|_{j}$, for $n=8$. These are compared against a numerical simulation on 500 submatrices of Haar-random matrices. The precise behaviour of this function is discussed in the supplemental material. Already for $n=8$, some points reach the value obtained in the limit of large $n$.

Given this result, we can write $\Delta P_{k} / P$ as a geometric series: $\Delta P_{k} / P_{0}=\sqrt{\sum_{j=k+1}^{n}\left(\left|c_{j}\right| x^{j}\right)^{2}}$. If we take the limit $n \rightarrow \infty$, this has the finite value:

$$
\Delta P_{k} / P_{0}=x^{k+1} / 2\left(1-x^{2}\right)^{1 / 2} .
$$

Our algorithm for simulating boson sampling with partially distinguishable photons is now as follows: given the desired accuracy of the simulation and the level of indistinguishability with which the expeirment is performed, use eq. 4 to evaluate the required value of $k$. Next, compute eq. 2 up to the $k$-th term, and feed the computed value of $P_{k}$ into a classical sampling algorithm, such as the Metropolis-Hastings algorithm [24]. Such algorithms can generate a sample from a probability distribution, even if the number of possible outcomes is large [25], which means that the number of modes does not enter into the hardness of boson sampling in general [26].

Finally, we detemine the regions of the parameter space where our algorithm is efficient. The solid lines in Figure 4 show the values of $n$ and $x$ for which eq. 4 has solutions for any $k<n$. These lines define a threshold which must be exceeded for the complexity of the problem not to scale polynomially with the total number of photons. State-ofthe-art supercomputers can compute permanents of size 50 in approximately an hour [27], which might therefore be taken as an estimate of the number of photons required to obtain a quantum advantage. Using eq. 4, we 


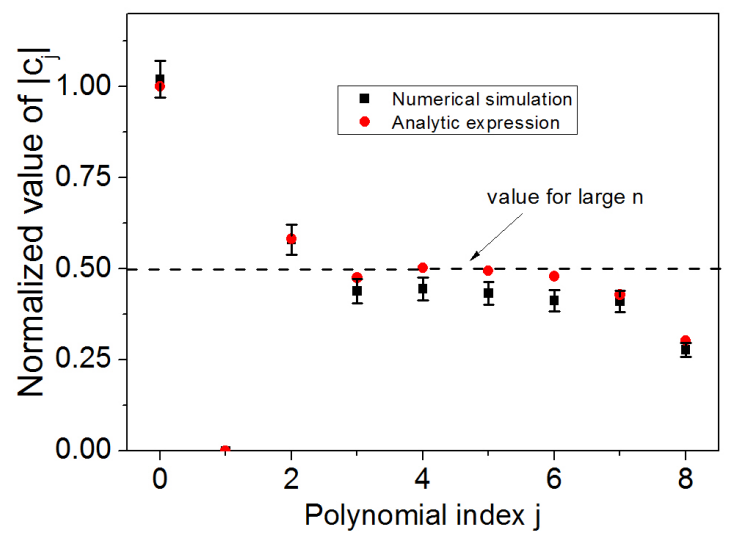

Figure 3. Coefficients of the polynomial $P=\sum_{j=0}^{n} c_{j} x^{j}$, for $n=8$. The black squares correspond to a numerical simulation of 500 random unitaries. The red circles correspond to the prediction from eq 4 .

find that we require $x=0.870, x=0.908$ and $x=0.947$ for $P_{49}$ to be accurate to within $0.1 \%, 1 \%$ or $10 \%$, respectively. Note that if we desire higher accuracy, our approximation fails at lower values of $x$. We stress again that this result is a lower bound: achieving these numbers in an experiment is no guarantee that the experiment is not classically simulable through some more advanced algorithm.

The dashed lines in Figure 4 show below which $n$ photon interference can be expressed as $n-1$ photon interference, to within the given accuracy (i.e. taking only the first term in the geometric series for $\Delta P / P)$. The area in between the dashed and solid lines is the region of parameter space where our approximation works for a finite value of $n$, but where the approximation will eventually fail at large enough $n$.

Finally, we consider the prefactor of our algorithm. The original expression from [16] requires the computation of roughly $2^{n}$ permanents of size $n$, whereas the original boson sampling proposal requires the computation of only one such permanent. If we limit the number of computations steps we allow ourselves for computing $P_{k}$ to be equal to the number of steps required to compute an $n$-by- $n$ permanent, we arrive at the dash-dotted curves in Figure 4. The area in between the solid and dash-dotted curves represents the region of the parameter space where the computational cost scales polynomially in the number of photons, but where our approximation might be impractical for small values of $n$. Since Tichy's expression requires the computation of many very similar permanents, we expect that there is significant scope for improvement on the classical algorithm at this point, which we leave as an open problem.

Finally, we note that our results do not depend on the original, fairly arbitrary parametrization of $S$. In particu-

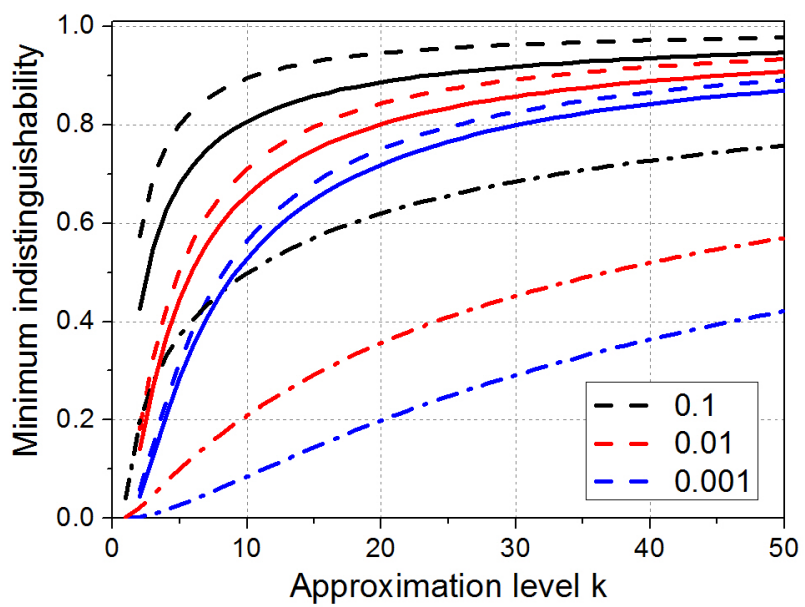

Figure 4. Lower bound on the quality of photons required to achieve exponential scaling of the hardness, as a function of the level of approxmation. The solid lines indicate solutions of eq.4 for and demarcate the region of polynomial scaling in $n$. The dashed lines indicate the value of $x$ below which $n$ photon interference can be expressed as interference of $n-1$ photons, The dash-dotted lines indicate the values of indistinguishability where $n$-photon interference can be so described using fewer resources than required for the computation of an $n$-by- $n$ complex permanent.

lar, if one has $S_{i j}=x_{i j}+\delta_{i j}\left(1-x_{i j}\right)$, with $x_{i j}<1$, one can apply the same expansion. A similar argument applies if the $x_{i j}$ are complex. In fact, the algorithm can be refined to first compute those terms with large $x_{i j}$, opening up the possibility of further approximations. Therefore our results apply in the experimentally relevant case where all photons are not of equal distinguishability.

In summary, we have shown how the limited indistinguishability of photons affects the hardness of the boson sampling problem. We have presented a scheme that can express the probability of an outcome in boson sampling as a sum of smaller permanents when the photons are sufficiently distinguishable. We have demonstrated that this scheme scales polynomially in the overall photon number, while its accuracy does not depend on photon number. We have used this scheme to estimate a lower bound on the indistinguishability required to achieve a quantum advantage.

We thank Raul Garcia-Patron and Nathan Walk for useful discussions. JJR is supported by NWO Rubicon. AM is supported by the Buckee Scholarship from Merton College, Oxford. GT is supported by the Merton Scholarship Fund. W.R.C. and I.A.W. acknowledge an ERC Advanced Grant (MOQUACINO). W.S.K. is supported by EPSRC EP/M013243/1. I.A.W. acknowledges the H2020-FETPROACT-2014 project QUCHIP (G.A. 641039). 
* jelmer.renema@physics.ox.ac.uk

[1] S. Aaronson and A. Arkhipov, Theory Comput. 9, 143 (2013).

[2] J. B. Spring et al., Science 339, 798 (2012).

[3] M. A. Broome et al., Science 339, 794 (2012).

[4] A. Crespi et al., Nat. Photon. 7, 545 (2013).

[5] M. Tillmann et al., Nat. Photon. 7, 540 (2013).

[6] M. Bentivegna et al., Science Advances 1, e1400255 (2015).

[7] J. Carolan et al., Science 349, 711 (2015).

[8] H. Wang et al., Nat. Photon. 11, 361 (2017).

[9] V. S. Shchesnovich, Phys. Rev. A 91 (2015).

[10] A. Arkhipov, Phys. Rev. A 92, 062326 (2015).

[11] S. Aaronson, Scattershot bosonsampling: a new approach to scalable bosonsampling experiments.

[12] A. P. Lund et al., Phys. Rev. Lett. 113, 100502 (2014).

[13] S. Rahimi-Keshari, T. C. Ralph, and C. M. Caves, Phys. Rev. X 6, 021039 (2016).
[14] V. S. Shchesnovich, Phys. Rev. A 89 (2014).

[15] V. S. Shchesnovich, Phys. Rev. A 91 (2015).

[16] M. C. Tichy, Phys. Rev. A 91, 022316 (2015).

[17] P. P. Rohde, Phys. Rev. A 91 (2015).

[18] V. Tamma and S. Laibacher, Quant. Inform. Proc. 15, 1241 (2015).

[19] V. Tamma and S. Laibacher, Phys. Rev. Lett. 114 (2015).

[20] S. Laibacher and V. Tamma, (2017), arXiv:1706.05578

[21] M. Tillmann et al., Phys. Rev. X 5 (2015).

[22] M. Jerrum, A. Sinclair, and E. Vigoda, A polynomialtime approximation algorithm for the permanent of a matrix with non-negative entries, in Proceedings of the thirty-third annual ACM symposium on Theory of computing - STOC '01, ACM Press, 2001.

[23] H. J. Ryser, Combinatorial Mathematics (Mathematical Association of America, 1963).

[24] W. K. Hastings, Biometrika 57, 97 (1970).

[25] L. Martino and V. Elvira, (2017), arXiv:1704.04629.

[26] A. Neville et al., No imminent quantum supremacy by boson sampling, 2017, arXiv:1705.00686.

[27] J. Wu et al., 2016, arXiv:1606.05836. 